\title{
FACTORES QUE INFLUYEN EN EL RENDIMIENTO DEL ALUMNADO DE LA ESO SEGÚN LA ELECCIÓN DE LA RAMA DE CIENCIAS O LETRAS
}

\author{
Jesús Barbado Yllas \\ Colegio SEI "Virgen de Soledad" (España) \\ Margarita Alcaide Risoto \\ Universidad Camilo José Cela (España) \\ Nuria Cantero Rodríguez \\ CEIP Navas de Tolosa (España)
}

\begin{abstract}
Resumen. Este artículo tiene como objetivo comprobar cuáles son los factores que tienen una mayor influencia en los alumnos que pasan de $3^{\circ}$ a $4^{\circ}$ de E.S.O. a la hora de elegir la rama de ciencias o letras. El estudio se realizó con una muestra de 23 alumnos. El instrumento utilizado fue el cuestionario de Autoconcepto AF-5 que contiene una batería de treinta preguntas y evalúa cinco dimensiones (Académico/Laboral, Social, Emocional, Familiar y Física). La educación es una de los pilares de cualquier sociedad civilizada. La formación académica debe ser acorde a las exigencias del mundo en el que vivimos. Esto hace que los alumnos deban tomar decisiones importantes desde edades tempranas. El análisis se centró en el autoconcepto y sus diferentes dimensiones y en cómo se relaciona con el género y el rendimiento académico. Se realizó un análisis comparativo de los datos utilizando el programa estadístico SPSS. Las conclusiones más importantes a las que se llegaron fueron que los alumnos con mayor autoconcepto académico tienen un rendimiento académico alto y la opción preferida por los alumnos con mayor rendimiento académico es la de ciencias. En cuanto al género, las mujeres tienen un mayor autoconcepto académico que los hombres. Además, no podemos decir que exista una relación entre el género y la opción elegida.
\end{abstract}

Palabras clave: autoconcepto, rendimiento académico, opción académica y género.

\section{FACTORS THAT INFLUENCE IN THE PERFORMANCE OF THE STUDENT OF THE ESO ACCORDING TO THE CHOICE OF THE SCIENCE OR LETTERS}

\begin{abstract}
This article aims to verify which are the factors that have a greater influence on students who pass from $3^{\circ}$ to $4^{\circ}$ of E.S.O. when it is time to choose between a Bachelor of sciences or a Bachelor of Arts. The study was carried out with a sample of 23 students. The instrument used was the AF-5 Selfconcept questionnaire that contains thirty questions and evaluates five aspects (Academic, Social, Emotional, Family and Physical). Education is one of the pillars of any civilized society. Academic formation must be in accordance with the demands of the world in which we live. This forces students to make important decisions from an early age. The analysis focused on self-concept and its different aspects and how it relates to gender and academic achievement. A comparative analysis of the data was performed by using the SPSS statistical program. The most important conclusions reached were that students with the higher academic self-concept have higher academic achievement and science is the preferred option for students with higher academic achievement. As for gender, women have greater academic self-concept than men. In addition, we cannot say that there is a relationship between gender and the option chosen.
\end{abstract}


Keywords: Self-concept, academic achievement, academic choice and gender.

\title{
FATORES QUE INFLUENCIAM NO RENDIMENTO POR ESTUDANTES DA ESO QUE ELEGEM A CARREIRA DE CIÊNCIAS OU LETRAS
}

\begin{abstract}
Resumo. Este artigo tem como objetivo verificar quais os fatores de maior influência sobre os estudantes que se deslocam do $3^{\circ}$ para o $4^{\circ}$ ano da E.S.O. ao escolher as áreas de Cências ou Letras. O estudo foi realizado com uma amostra de 23 alunos. O instrumento utilizado foi o questionário Autoconceito AF-5 que contém trinta perguntas e avalia cinco dimensões (Acadêmica/Laboral, Social, Emocional, Famíliar e Física). A educação é um dos pilares de qualquer sociedade civilizada. A formação acadêmica deve estar de acordo com as exigências do mundo em que vivemos. Isso faz com que os alunos devam tomar decisões importantes desde idades precoces. A análise centrou-se no autoconceito e suas diferentes dimensões e como se relaciona com o sexo e rendimento acadêmico. Uma análise comparativa dos dados foi realizada por meio do uso programa estatístico SPSS. As conclusões mais importantes indicam que os alunos com maior autoconceito acadêmico têm maior rendimento e a escolha preferida para os alunos com rendimento mais elevado é Ciências. Quanto ao sexo, as mulheres têm um autoconceito acadêmico mais elevado que os homens. Além disso, não podemos dizer que existe uma relação entre gênero e a opção escolhida.
\end{abstract}

Palavras-chave: autoconceito, drendimento acadêmico, opção acadEmica e gênero.

\section{Introducción}

\section{Introducción al problema}

Los seres humanos tenemos innata una capacidad que nos diferencia de otros seres vivos como los animales y las plantas. Esta aptitud innata se basa en la posibilidad de tomar decisiones entre diferentes alternativas existentes. Pero esto no es algo nuevo ni novedoso. Esta cualidad es tan antigua como la existencia de los propios seres humanos.

La toma de decisiones ha sido tratada a lo largo del tiempo desde diferentes campos, como pueden ser las Matemáticas, la Psicología o la Economía. Los matemáticos deben tomar decisiones sobre cuál es la mejor estrategia para resolver un problema. Los psicólogos estudian cómo puede afectar la conducta humana y nuestros comportamientos en las decisiones que tomamos a diario. Y los economistas tratan de buscar la mejor combinación posible de recursos y factores productivos para poder satisfacer las necesidades de los individuos. Estas decisiones tienen efecto en nuestra forma de visionar el mundo y en definitiva en nuestra satisfacción vital (Molero et al. 2013).

Pero nuestro ciclo vital está plagado de problemas de decisión. A medida que nos desarrollamos como seres humanos aumenta la dificultad en la toma de decisiones y nos hacemos más conscientes de las consecuencias que pueden tener una buena o mala decisión. Al principio, el instinto nos ayuda a elegir entre varias alternativas. Después, nuestras decisiones deben estar fundamentadas por conductas racionales.

La sociedad en la que vivimos demanda personas con liderazgo y que sean capaces de tomar decisiones en determinadas situaciones. Por eso, en la escuela debe 
fomentarse la toma de decisiones por parte de los alumnos, como un proceso formativo para el miembro social del mañana.

\section{Autoconcepto}

Muchas han sido las definiciones que han tratado de explicar el autoconcepto. Según Harter (1990), el autoconcepto alude a como el individuo se percibe. De la misma manera, Kalish (1983) explica que cada individuo tiene su propia imagen de sí mismo y que esta imagen está influenciada por las diferentes experiencias vividas.

A lo largo del tiempo, los modelos para analizar el autoconcepto han ido cambiando. Se ha pasado de unos modelos que consideraban el autoconcepto como algo que tenía una sola dimensión, capaz de evaluar el autoconcepto de forma global Harter (1990), a unos modelos que enfocaban el autoconcepto desde diferentes dimensiones o perspectivas (Shavelson, Hubner y Stanton (1976). Estos modelos multidimensionales evalúan el autoconcepto por separado, utilizando diferentes instrumentos para cada dimensión.

En las últimas dos décadas, la relación entre autoconcepto y género ha irrumpido con gran fuerza y muchos han sido los investigadores que han tratado de analizar la existencia o no de diferencias en el autoconcepto entre el género masculino y femenino. Por ejemplo, Miller (1979), afirma que el nivel de autoconcepto en las chicas es menor que en el de los chicos. Por el contrario, (Crain, 1996), sostiene que los resultados sobre la influencia del autoconcepto en chicos y chicas había sido contradictorio. Además, concluye que las posibles diferencias se centran en campos determinados, como, por ejemplo, el autoconcepto físico, no a nivel de autoconcepto general.

A continuación, se definen las 5 dimensiones del autoconcepto:

- $\quad$ Autoconcepto académico. Hace referencia a la percepción que el sujeto tiene de la calidad de su desempeño, como estudiante o como trabajador. Se centra en dos aspectos: el primero relativo al sentimiento que se genera en el estudiante o en el trabajador acerca de su actividad a través de sus profesores o superiores (buen alumno, buen trabajador...) y, el segundo, referido a cualidades concretas valoradas especialmente en ese contexto (inteligencia, hábito de trabajo...).

- Autoconcepto social. Percepción que el individuo tiene de su competencia en las relaciones sociales. Abarca dos aspectos: uno referido a la red social del sujeto y a su facilidad o dificultad para mantenerla o ampliarla; otro, perteneciente a algunas cualidades importantes en las relaciones interpersonales (amigable y alegre).

- Autoconcepto emocional. Es la captación que el sujeto tiene sobre su estado emocional y sus respuestas a situaciones específicas, con cierto grado de compromiso e implicación en su vida cotidiana. Permite diferenciar dos aspectos: el primero hace referencia a la percepción general sobre el propio estado emocional (me asusto con facilidad, estoy nervioso), y el segundo centrado en la autovaloración emocional en situaciones concretas (cuando me preguntan o me hablan) en las que la otra persona es de rango superior (profesor, jefe...).

- Autoconcepto familiar. Es la percepción que tiene el sujeto de su implicación, participación e integración en el medio familiar. Esta dimensión abarca dos aspectos. El primero de ellos específicamente a la confianza y el afecto de los padres. El segundo relativo a cuatro variables de la familia y el hogar. 
- Autoconcepto físico. Hace referencia a la percepción que tiene el sujeto de su aspecto físico y de su condición física. Esta dimensión incluye dos aspectos complementarios. Uno referido a la práctica deportiva en la vertiente social, física y de habilidad. Otro relativo al aspecto físico.

En conclusión, el autoconcepto es la imagen que cada individuo tiene de su propio ser y que dicha imagen se ve influenciada por las propias experiencias. No podemos analizar el autoconcepto como algo global, sino que se compone de diferentes dimensiones que afectan a los individuos de muy variadas formas.

\section{Rendimiento Académico}

Intentar definir el concepto de rendimiento académico resulta complicado, ya que existen múltiples factores que pueden influir en este aspecto educativo. Muchos son los autores que han hablado sobre este concepto, como Tournon (1984), que lo define como el fruto del aprendizaje, el cual ha sido promovido por el docente y llevado a cabo por el alumno. Por su parte, Jiménez (2000) afirma que el rendimiento académico es "el nivel de conocimientos demostrado en un área o materia comparado con la norma de edad y nivel académico".

No debemos pensar que el rendimiento académico es el resultado de una sola magnitud, sino que es la consecuencia o suma de una multitud de elementos que afectan a los alumnos. Algunos de estos elementos pueden ser las calificaciones obtenidas por los alumnos en pruebas objetivas, el nivel o factor cognitivo o el entorno socioeconómico en el que el alumno se desarrolla.

En consecuencia, podemos afirmar que son muchos los factores que intervienen en la consecución de los diferentes logros académicos, pero la confluencia de los factores escolares y los aspectos del contexto social del alumno serán los responsables, en mayor medida, de la obtención de un buen resultado en el rendimiento académico.

\section{Características del alumnado de E.S.O.}

Los alumnos de Educación Secundaria Obligatoria (E.S.O.) se caracterizan, entre otras, por verse inmersos en una etapa evolutiva llamada adolescencia. Esta etapa se desarrolla entre los 12 y los 20 años, aproximadamente, y, por tanto, supone una transición entre la niñez y la etapa adulta. La adolescencia destaca por los continuos y grandes cambios que se experimentan en muchos ámbitos o aspectos, como pueden ser aspectos físicos, cognitivos, afectivos y sociales.

A nivel conductual y emocional, la adolescencia y sus cambios hormonales puede ser la causante de continuas variaciones en el estado de ánimo y cambios de humor (Brooks-Gunn, Graber y Paikoff, 1994). Dichos cambios hormonales pueden reflejarse de manera distinta según el sexo, como por ejemplo conductas agresivas en los chicos y estados depresivos en las chicas (Buchanan, Maccoby y Dornbusch, 1992; Steinberg y Silk, 2002).

En el ámbito cognitivo se experimenta un gran cambio, debido a las variaciones que se producen en su forma de pensar y razonar. Según Piaget (1973), los alumnos entran en un estadío llamado "pensamiento formal", el cual les permite razonar de una forma más lógico e incrementar su poder de abstracción. Una de las consecuencias de esta forma de pensar y razonar es el pensamiento crítico, el cual causará que el adolescente ponga en duda las normas que hasta entonces había seguido sin ninguna objeción. Esto hecho llevará consigo un aumento de los conflictos en sus relaciones familiares y sociales (Smetana, 1989). 
Otro de los aspectos que va a marcar la etapa adolescente es la construcción y consolidación de la personalidad. La adolescencia es una etapa de búsqueda continua de nuevas sensaciones que culminen en la construcción de la identidad futura a nivel personal y profesional. Muchas de las experiencias vividas en esta etapa de construcción de la personalidad están favorecidas por la escasa aversión al riesgo inherente a esta edad (Chambers, Taylor y Potenza, 2003).

\section{Estudios sobre factores que pueden influir en los alumnos en la toma de decisiones a la hora de elegir ciencias o letras.}

En el presente estudio hemos intentado reflejar la multidimensionalidad de las decisiones que, ya en la etapa de la E.S.O., deben tomar los alumnos. Prueba de ello, son la variedad de factores que pueden afectar a las elecciones académicas en esta etapa, y que, sin duda, afectarán a su futura vida profesional (Goñi et al, 2007).

En este sentido, Osipow (1982), clasifica los factores influyentes en las decisiones académicas de los alumnos como "Aptitudes profesionales, Intereses profesionales, Personalidad, Realización, Hogar y familia, Economía e Impedimentos".

Por su parte Castaño (1983), intenta clasificar y dar nombre a diversos ámbitos que puedan englobar a todos los factores que puedan afectar a los alumnos. En primer lugar, este autor identifica factores que implican situaciones naturales y sociales. De la misma manera, Castaño engloba dentro de otro ámbito todos aquellos factores relacionados con la personalidad, la motivación la cognición de cada alumno. En el tercer ámbito, clasifica todos los factores relacionado con las experiencias personales. Finalmente, en el cuarto y último ámbito, se recogen factores que tienen que ver con la probabilidad de éxito.

También nos parece muy importante la propuesta de clasificación de los diferentes factores que realizan Royo y Jiménez Correa (1993). Para ellos, los factores se pueden clasificar en factores socioeconómicos (recogen lo concerniente a las características personales y familiares de los alumnos y a su contexto social), factores psicológicos (todas aquellas aptitudes, autoconcepto, motivaciones, intereses y valores que afectan en la toma de decisiones de los alumnos), factores pedagógicos (basados en el rendimiento académico de los alumnos y todas aquellas acciones de orientación que los alumnos hayan experimentado en el centro escolar) y factores institucionales (donde se recoge las características de los centros educativas y los condicionantes legales y curriculares que puedan surgir).

\section{Objetivos de la Investigación}

El objetivo general de esta investigación será comprobar la posible relación o grado de asociación entre la opción elegida (ciencias o letras) y las distintas dimensiones del autoconcepto (académico, físico, social, emocional, familiar) y rendimiento académico de los alumnos de $3^{\circ}$ de la Educación Secundaria Obligatoria.

Además, se proponen una serie de objetivos específicos:

- Comprobar si existe relación entre el autoconcepto total y la opción elegida.

- Comprobar si existe relación entre la variable género y los distintos autoconceptos.

- Comprobar si existe relación entre el autoconcepto total y el rendimiento académico. 


\section{Hipótesis}

López-Baraja (1985) viene a determinar que la hipótesis es la etapa fundamental en la investigación, convirtiéndose en el instrumento más valioso del trabajo. Efectivamente, de las hipótesis derivan las variables de estudio, el campo de investigación, información a recoger, métodos a emplear y por supuesto, las interpretaciones sobre los resultados.

En función de los objetivos planteados se definen las siguientes hipótesis:

- Hipótesis H1. Los alumnos que han elegido ciencias tienen un autoconcepto académico, social, físico, familiar, emocional y un rendimiento académico más alto que aquellos alumnos que han elegido letras.

- Hipótesis H2. Los alumnos que tienen un autoconcepto total alto han elegido ciencias.

- Hipótesis H3. Las mujeres tienen un autoconcepto en todas sus dimensiones más alto que los hombres.

- Hipótesis H4. Los alumnos con un autoconcepto total alto tienen un rendimiento académico alto.

\section{Método}

\section{Diseño y tipo de Investigación}

De acuerdo con los objetivos planteados, el estudio sigue el método descriptivo, realizando un análisis comparativo en base a los datos recogidos. También se realiza una correlación de Pearson para saber la significatividad entre los distintos autoconceptos, el género, el rendimiento y la opción elegida de los alumnos de secundaria.

\section{Participantes}

El estudio se realizó en el Colegio SEI Virgen de la Soledad situado en la localidad de Arganda del Rey (Madrid). Es un centro concertado. El curso en el que se llevó a cabo la investigación fue en $3^{\circ}$ de la ESO. Se le pasó el cuestionario a 23 alumnos, los cuales participaron voluntariamente. De los 23 alumnos, 11 eran chicos y 12 eran chicas.

\section{Instrumentos}

Para poder llevar a cabo la investigación se ha utilizado el cuestionario Autoconcepto Forma 5, desarrollado por García y Musitu (2001). Tras el análisis del contexto (tipo de centro y alunando), se decide usar este instrumento como el más apropiado puesto que mide cinco dimensiones básicas del autoconcepto en niños, adolescentes y adultos, estas cinco dimensiones son las siguientes: académico/profesional, social, emocional, familiar y físico. Cada una de las dimensiones consta de 6 ítems, es decir; hacen un total de 30 cuestiones las cuales los individuos deben de contestar utilizando una escala del 1 a los 99 puntos, siendo 1 totalmente en desacuerdo y 99 totalmente de acuerdo. Esta forma de respuesta permite dar cuenta del problema de la baja discriminación en las puntuaciones altas, situación común en las pruebas de autoconcepto (Tomás y Oliver, 2004). 
Se comprueba análisis de validez factorial y confiabilidad de la escala, siguiendo a Riquelme (2011), utilizando el programa SPSSS V.16, AMOS 6.1 y LISREL 8.8. En primer lugar, para el estudio de la validez factorial del cuestionario, se utilizó un análisis factorial confirmatorio (AFC), partiendo del modelo a priori de la estructura propuesta por García y Musitu (1999). A través del AFC se pretendió observar en qué medida el modelo teórico de cinco factores propuesto en el instrumento se ajusta a los datos utilizados. La confiabilidad se ha medido a través del alfa de Cronbach, en la que se observa la coherencia general de la prueba, y particularmente describiendo en qué medida las respuestas altas coinciden con las altas y las bajas con las bajas en todos los ítems (Aron y Aron, 2001). En este análisis se calculó el alfa de las saturaciones factoriales del AFC, la relación ítem-factor.

Para medir el rendimiento académico se utilizó las calificaciones obtenidas por los alumnos a lo largo de los tres primeros cursos de Educación Secundaria Obligatoria (E.S.O.)

\section{Variables}

A continuación, se describen y clarifican las variables que intervienen en nuestro estudio:

- Variables principales: autoconcepto y rendimiento académico.

- Variables Complementarias: opción y género.

\section{Procedimiento}

El cuestionario se llevó a cabo en el Colegio Concertado "SEI Virgen de la Soledad" situado en la localidad de Arganda del Rey (Madrid), bajo la previa autorización del equipo directivo. Realizamos el cuestionario Autoconcepto Forma 5, a los alumnos de $3^{\circ}$ de ESO, a primera hora de la mañana.

Hicimos una breve explicación de cómo puntuar las diferentes frases de 0 a 99 puntos. Con este objetivo y de manera grupal, realizamos varios ejemplos lanzando diferentes preguntas al aire para que los alumnos lo entendieran. Después, una vez asegurados que todos los participantes lo habían entendido, de forma individual realizaron el cuestionario.

Posteriormente, el análisis estadístico y la obtención de resultados fue realizado mediante el programa SPSS, versión 20.0, y que a continuación mostramos.

\section{Resultados}

\section{Autoconcepto Académico - Opción elegida}

Vamos a tratar de observar cuál es la relación existente entre el autoconcepto académico y la opción elegida. Con este fin, utilizaremos la tabla 1, en la que se puede observar la media de la puntuación obtenida en el autoconcepto académico según la opción elegida. 
Tabla 1

Autoconcepto académico y opción elegida

\begin{tabular}{crc}
\hline Autoconcepto académico & Ciencias \% & \multicolumn{1}{c}{ Letras \% } \\
\hline $\mathbf{- 5 0}$ & 11,1 & 63,6 \\
\hline $\mathbf{+ 5 0}$ & 88,9 & 36,4 \\
\hline
\end{tabular}

Nota: elaboración propia

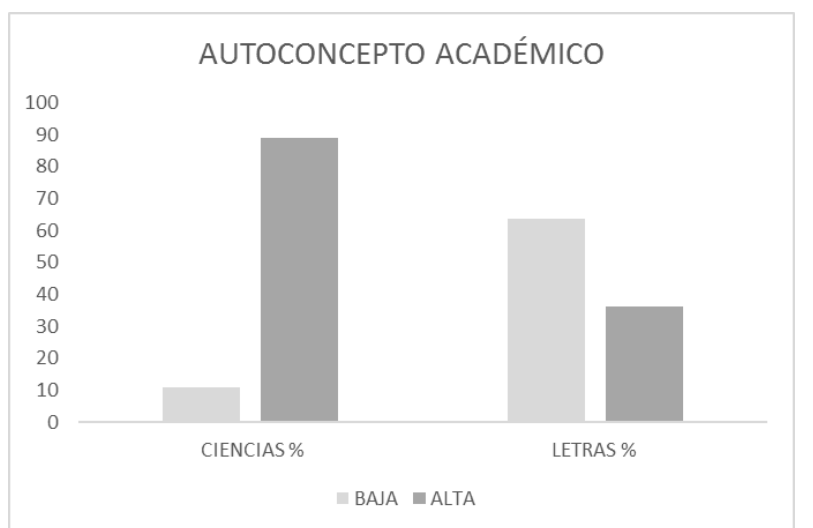

Figura 1. Autoconcepto académico y opción elegida

Como podemos observar en la tabla 1, así como en la figura 1, que el 88,9\% de las personas que eligieron ciencias tenían un autoconcepto académico alto respecto al $11,1 \%$ de las personas que lo tenían bajo. En el caso de letras, el 36,4\% de las personas que escogieron esta opción tenían un autoconcepto alto frente al 63,6\% que lo tenían bajo. Por lo que los alumnos con autoconcepto académico alto se inclinan más por las ciencias que por las letras.

\section{Autoconcepto Social - Opción elegida}

Vamos a tratar de observar cuál es la relación existente entre el autoconcepto social y la opción elegida. Con este fin, utilizaremos la tabla 2, en la que se puede observar la media de la puntuación obtenida en el autoconcepto social según la opción elegida.

Tabla 2

Autoconcepto social y opción elegida

\begin{tabular}{crc}
\hline Autoconcepto social & Ciencias \% & \multicolumn{1}{c}{ Letras \% } \\
\hline $\mathbf{- 5 0}$ & 33,3 & 36,4 \\
\hline $\mathbf{+ 5 0}$ & 66,7 & 63,6 \\
\hline
\end{tabular}

Nota: elaboración propia 


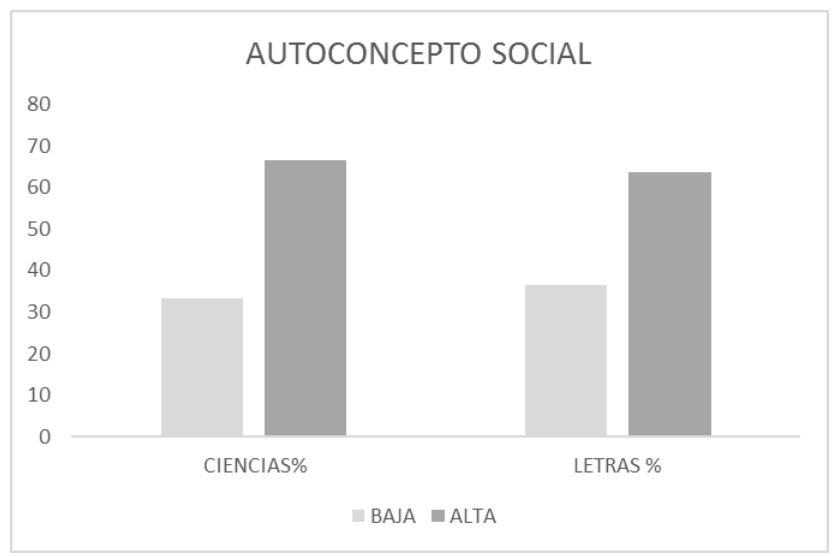

Figura 2. Autoconcepto social y opción elegida

Podemos observar en la tabla y figura 2 que el $66,7 \%$ de las personas que eligieron ciencias tenían un autoconcepto social alto respecto al 33,3\% de las personas que lo tenían bajo. En el caso de letras, el $36,4 \%$ de las personas que escogieron esta opción tenían un autoconcepto bajo frente al $63,6 \%$ que lo tenían alto. Por lo que en este caso podemos decir que el autoconcepto social no es un factor significativo en la opción elegida, ya que los alumnos con un autoconcepto social alto eligen indistintamente ciencias o letras.

\section{Autoconcepto Emocional - Opción elegida}

Vamos a tratar de observar cuál es la relación existente entre el autoconcepto emocional y la opción elegida. Con este fin, utilizaremos la tabla 3, en la que se puede observar la media de la puntuación obtenida en el autoconcepto emocional según la opción elegida.

Tabla 3.

Autoconcepto Emocional y opción elegida

\section{Autoconcepto emocional}

\begin{tabular}{lll}
\hline $\mathbf{- 5 0}$ & 44,4 & 36,4 \\
\hline $\mathbf{+ 5 0}$ & 55,6 & 63,6 \\
\hline
\end{tabular}

Nota: elaboración propia

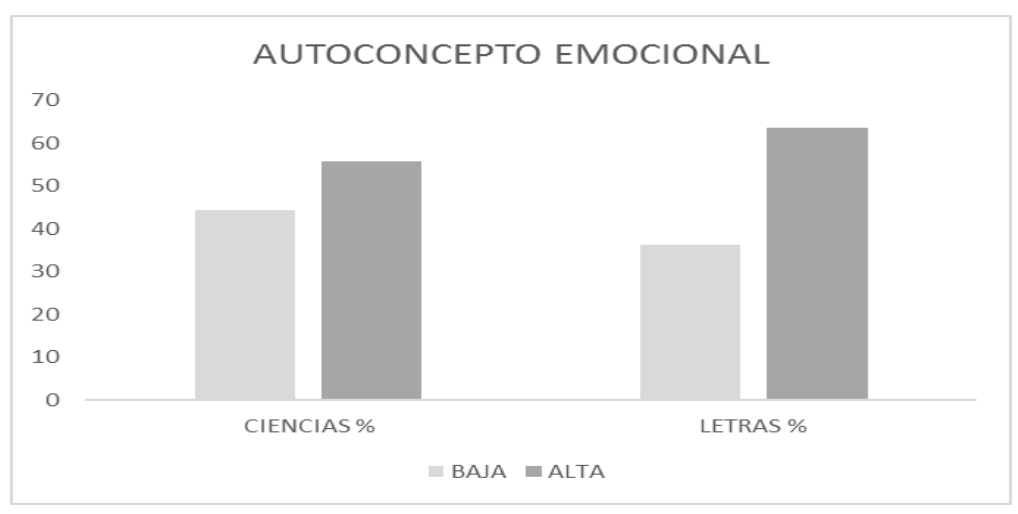

Figura 3. Autoconcepto emocional y opción elegida

\section{Letras \%}


Se observa en la tabla y figura 3 que el 55,6\% de las personas que eligieron ciencias tenían un autoconcepto emocional alto respecto al 44,4\% de las personas que lo tenían bajo. En el caso de letras, el 36,4\% de las personas que escogieron esta opción tenían un autoconcepto bajo frente al 63,6\% que lo tenían alto. Por tanto, podemos concluir que las personas con un autoconcepto emocional alto escogen la opción de letras.

\section{Autoconcepto Familiar - Opción elegida}

Vamos a tratar de observar cuál es la relación existente entre el autoconcepto familiar y la opción elegida. Con este fin, utilizaremos la tabla 4, en la que se puede observar la media de la puntuación obtenida en el autoconcepto familiar según la opción elegida

Tabla 4

Autoconcepto Familiar y opción elegida

\begin{tabular}{clc}
\hline Autoconcepto familiar & Ciencias \% & Letras \% \\
\hline $\mathbf{- 5 0}$ & 33,3 & 36,4 \\
\hline $\mathbf{+ 5 0}$ & 66,7 & 63,6 \\
\hline
\end{tabular}

Nota: elaboración propia

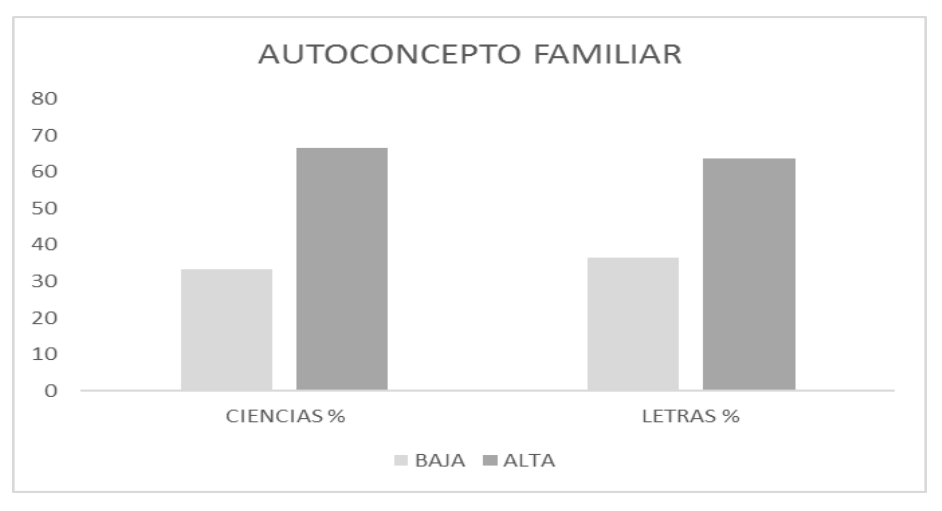

Figura 4. Autoconcepto familiar

Como podemos observar en la tabla y figura 4 , el $66,7 \%$ de las personas que eligieron ciencias tenían un autoconcepto familiar alto respecto al 33,3\% de las personas que lo tenían bajo. En el caso de letras, el 36,4\% de las personas que escogieron esta opción tenían un autoconcepto bajo frente al $63,6 \%$ que lo tenían alto. En este caso podemos decir que el autoconcepto familiar no influye significativamente en la opción elegida, ya que los alumnos con un autoconcepto familiar alto eligen indistintamente ciencias o letras.

\section{Autoconcepto Físico - Opción elegida}

Vamos a tratar de observar cuál es la relación existente entre el autoconcepto físico y la opción elegida. Con este fin, utilizaremos la tabla 5, en la que se puede 
observar la media de la puntuación obtenida en el autoconcepto físico según la opción elegida

Tabla 5

Autoconcepto Físico y opción elegida

\begin{tabular}{ccc}
\hline Autoconcepto físico & Ciencias \% & Letras \% \\
\hline $\mathbf{- 5 0}$ & 33,3 & 27,3 \\
$\mathbf{+ 5 0}$ & 66,7 & 72,7
\end{tabular}

Nota: elaboración propia

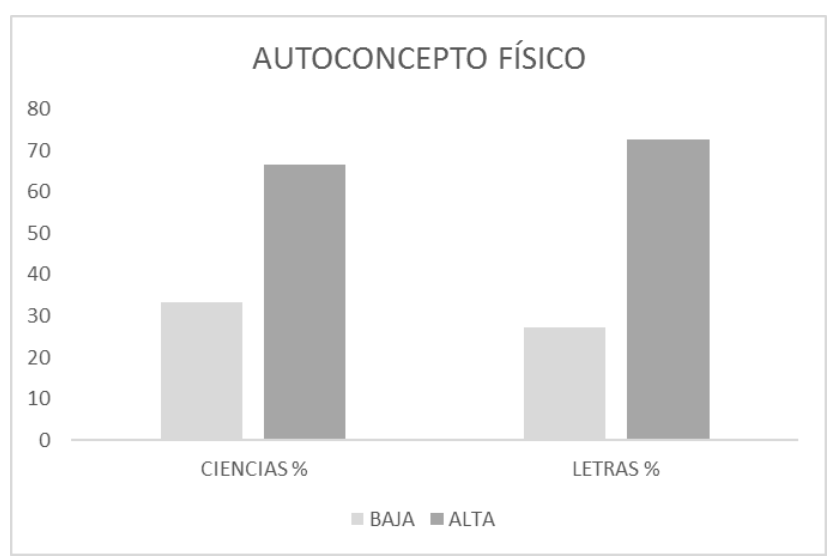

Figura 5. Autoconcepto físico y opción elegida

Se observa en la tabla 5, así como en la figura 5, que el $66,7 \%$ de las personas que eligieron ciencias tenían un autoconcepto físico alto respecto al 33,3\% de las personas que lo tenían bajo. En el caso de letras, el $72,7 \%$ de las personas que escogieron esta opción tenían un autoconcepto alto frente al 27,3\% que lo tenían bajo. Por lo que los alumnos con autoconcepto físico alto se inclinan más por las letras que por las ciencias.

\section{Autoconcepto Total - Opción elegida}

En este caso, trataremos de observar qué relación existe entre el autoconcepto total y la opción elegida y cómo, dependiendo de si tu autoconcepto es alto o bajo eliges ciencias o letras. Para ello, vamos a utilizar la tabla 6, en la que observamos la media de la puntuación obtenido en el autoconcepto total según la opción elegida.

Tabla 6

Autoconcepto Total y opción elegida

\begin{tabular}{ccc}
\hline Autoconcepto total & Ciencias \% & Letras \% \\
\hline $\mathbf{- 5 0}$ & 22,2 & 18,18 \\
\hline $\mathbf{+ 5 0}$ & 77,8 & 81,82 \\
\hline
\end{tabular}

Nota: elaboración propia 


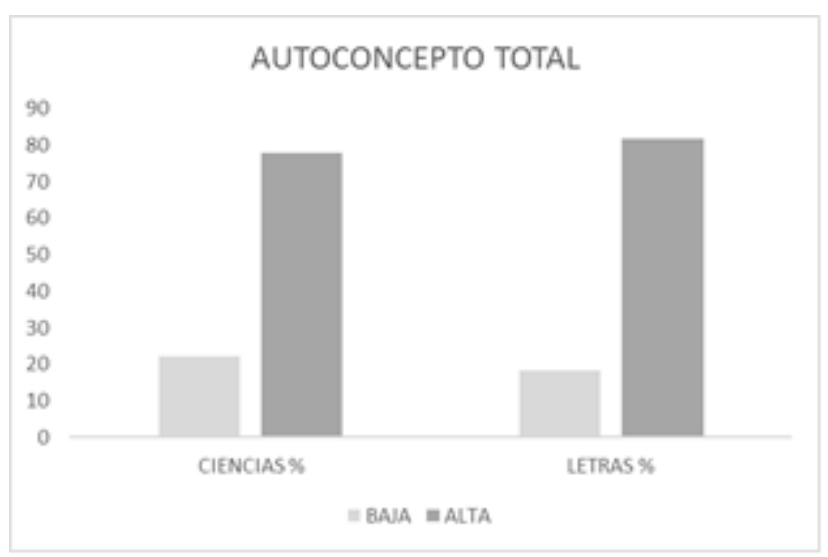

Figura 6. Autoconcepto total y opción elegida

Como podemos observar en la tabla 6 , así como en la figura 6 , el 77,8\% de las personas que eligieron ciencias tenían un autoconcepto alto respecto al 22,2\% de las personas que lo tenían bajo. En el caso de letras, el $81,82 \%$ de las personas que escogieron esta opción tenían un autoconcepto alto frente al $18,18 \%$ que lo tenían bajo. Por lo que los alumnos con autoconcepto total alto se inclinan más por las letras que por las ciencias.

\section{Autoconcepto Académico - Género}

En este apartado vamos a tratar de determinar que género tiene un autoconcepto académico más alto. Para ello vamos a utilizar la tabla 7, en la que podremos observar la media de la puntuación obtenida en el autoconcepto académico de cada género.

Tabla 7

Autoconcepto Académico y género

\begin{tabular}{lll}
\hline Autoconcepto académico & Hombre \% & Mujer \% \\
\hline $\mathbf{- 5 0}$ & 55,5 & 27,3 \\
\hline $\mathbf{+ 5 0}$ & 44,5 & 72,7 \\
\hline
\end{tabular}

Nota: elaboración propia

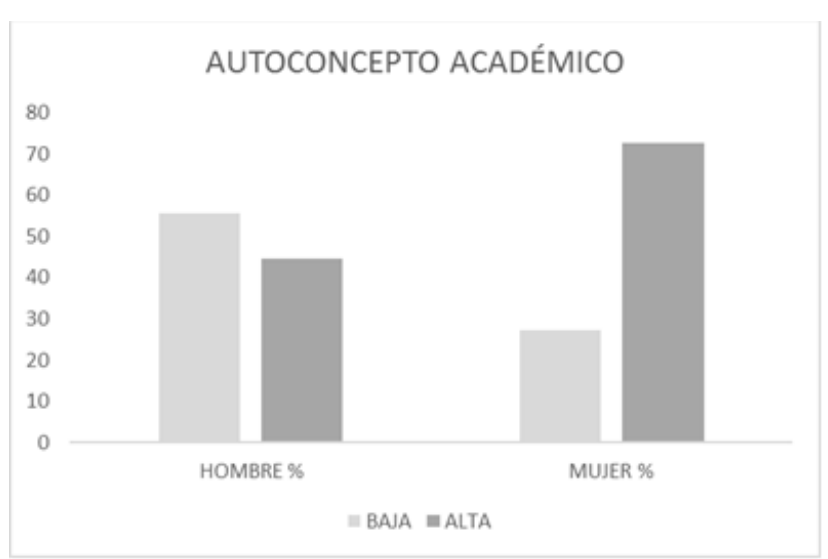

Figura 7. Autoconcepto académico y género 
Se puede observar en la tabla 7 y en la figura 7, que las mujeres tienen una media mayor en su autoconcepto académico que los hombres. De las 11 mujeres que participaron en el estudio, 8 tienen una puntuación mayor de 50, o lo que es lo mismo el $72,7 \%$, mientras que, de los 9 hombres participantes, solo 4 tuvieron una puntuación de más de 50 , lo que equivaldría a un 44,5\%.

\section{Autoconcepto Social - Género}

El siguiente apartado tratar de describir que género tiene un autoconcepto social más alto. Para ello vamos a utilizar la tabla 8 , en la que podremos observar la media de la puntuación obtenida en el autoconcepto social de cada género.

Tabla 8

Autoconcepto Social y género

\begin{tabular}{lll}
\hline Autoconcepto social & Hombre \% & Mujer\% \\
\hline $\mathbf{- 5 0}$ & 22,2 & 45,5 \\
\hline $\mathbf{+ 5 0}$ & 77,8 & 54,5 \\
\hline
\end{tabular}

Nota: elaboración propia

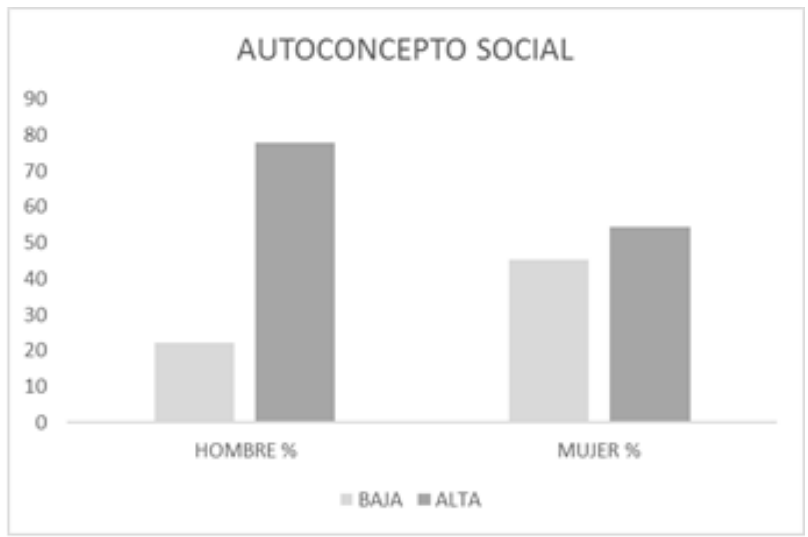

Figura 8. Autoconcepto social y género

La tabla y la figura 8 refleja que los hombres tienen una media mayor en su autoconcepto social que las mujeres. Es decir, de 9 hombres, 7 de ellos obtienen una puntuación mayor de 50 lo que correspondería al 77,8\%, mientras que en las mujeres tan solo 6, de las 11 mujeres participantes, tienen un autoconcepto total mayor de 50, lo que equivaldría al $54,5 \%$.

\section{Autoconcepto Emocional - Género}

Este punto trata de describir que género tiene un autoconcepto emocional más alto. Para ello vamos a utilizar la tabla 9, en la que podremos observar la media de la puntuación obtenida en el autoconcepto emocional de cada género. 
Tabla 9

Autoconcepto Emocional y género

\begin{tabular}{ccc}
\hline Autoconcepto emocional & Hombre \% & Mujer \% \\
\hline $\mathbf{- 5 0}$ & 33,3 & 45,5 \\
\hline $\mathbf{+ 5 0}$ & 66,7 & 54,5 \\
\hline
\end{tabular}

Nota: elaboración propia

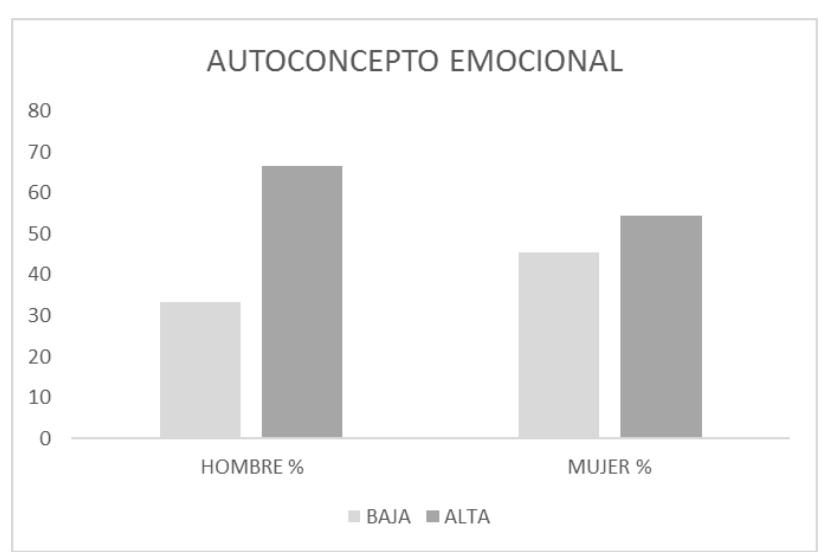

Figura 9. Autoconcepto Emocional y género

La tabla y la figura 9 refleja que los hombres tienen una media mayor en su autoconcepto emocional que las mujeres. De los 9 hombres que participaron en el estudio, 6 tienen una puntuación mayor de 50, o lo que es lo mismo el 66,7\%, mientras que, de las 11 mujeres participantes, 6 tuvieron una puntuación de más de 50, lo que equivaldría a un $54,5 \%$.

\section{Autoconcepto Familiar - Género}

En este apartado vamos a tratar de determinar que género tiene un autoconcepto familiar más alto. Para ello vamos a utilizar la tabla 10, en la que podremos observar la media de la puntuación obtenida en el autoconcepto familiar de cada género.

Tabla 10

Autoconcepto Familiar y género

\begin{tabular}{crc}
\hline Autoconcepto familiar & Hombre \% & Mujer \% \\
\hline $\mathbf{- 5 0}$ & 33,3 & 36,4 \\
\hline $\mathbf{+ 5 0}$ & 66,7 & 63,6 \\
\hline
\end{tabular}

Nota: elaboración propia 


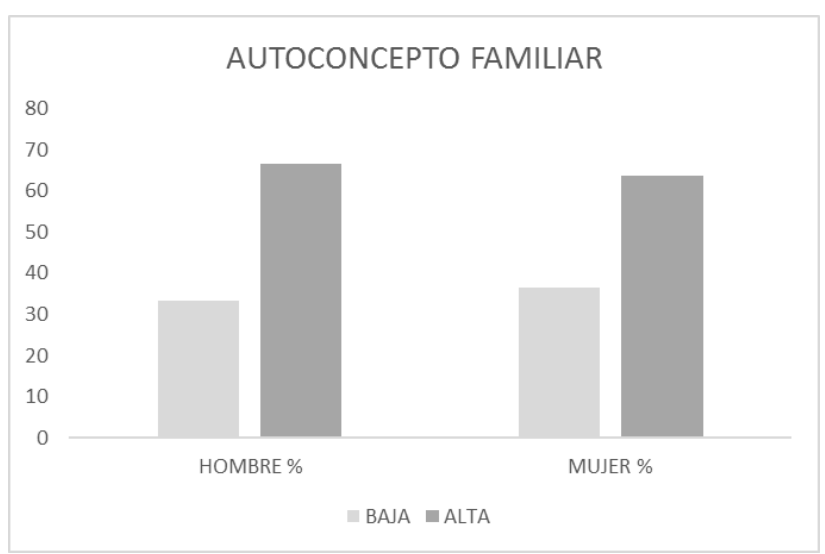

Figura 10. Autoconcepto Familiar y género

Como podemos observar en la tabla y figura 10, los hombres tienen una media ligeramente mayor en su autoconcepto familiar que las mujeres. El 66,7\% de los hombres tienen un autoconcepto familiar mayor de 50, en comparación al 63,6\% de las mujeres, que también tienen una puntuación mayor de 50 en su autoconcepto familiar.

\section{Autoconcepto Físico - Género}

El siguiente apartado pretende determinar que género tiene un autoconcepto físico más alto. Para ello se utiliza la tabla 11, en la que podremos observar la media de la puntuación obtenida en el autoconcepto físico de cada género.

\section{Tabla 11}

Autoconcepto Físico y género

\begin{tabular}{lll}
\hline Autoconcepto físico & Hombre \% & Mujer \% \\
\hline $\mathbf{- 5 0}$ & & 36,4 \\
\hline $\mathbf{+ 5 0}$ & 22,2 & 63,6 \\
\hline
\end{tabular}

Nota: elaboración propia

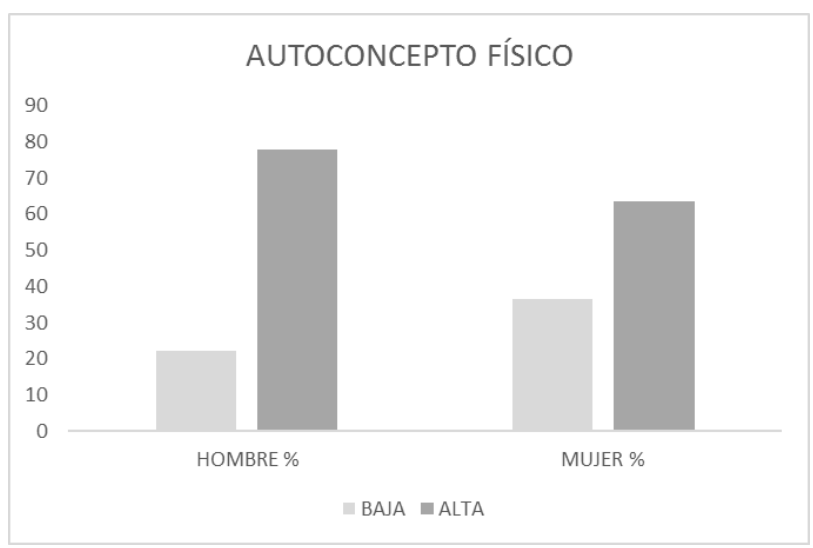

Figura 11. Autoconcepto físico y género 
Observamos en la tabla y figura 11, que los hombres obtienen una media mayor respecto a las mujeres en el autoconcepto físico. De hecho, de 9 hombres, 7 de ellos obtienen una puntuación mayor de 50 lo que correspondería al 77,8\%, mientras que en las mujeres tenemos 7 de 11 que tengan un autoconcepto físico mayor de 50, lo que equivaldría al $63,6 \%$.

\section{Autoconcepto Total - Rendimiento Académico}

En el siguiente apartado queremos observar si el nivel de autoconcepto total está relacionado con el rendimiento académico. Para ello se utiliza la tabla 13, en la que podremos observar la media de la puntuación obtenida en el autoconcepto total según el correspondiente rendimiento académico.

Tabla 12

Autoconcepto Total y Rendimiento Académico

\begin{tabular}{rccccc}
\hline A.total & Susp. \% & Aprob. \% & Not \% & Sob \% \\
\hline $\mathbf{- 5 0}$ & 0 & 15 & 5 & 0 \\
\hline $\mathbf{+ 5 0}$ & 15 & 30 & 30 & 5 \\
\hline
\end{tabular}

Nota: elaboración propia

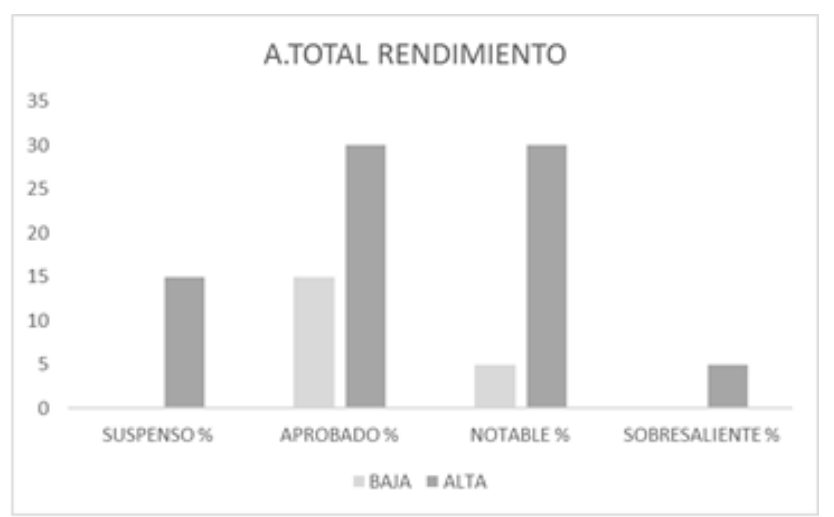

Figura 12. Autoconcepto total y Rendimiento académico

Como podemos observar en la tabla y la figura 12, aquellos alumnos que presentan un autoconcepto total alto obtienen un rendimiento académico superior a los que presentan un autoconcepto total bajo.

Existe una correlación significativa entre el autoconcepto académico y el rendimiento académico y entre el autoconcepto académico y la opción elegida. No ocurre lo mismo con los otros tipos de autoconcepto.

\section{Discusión y conclusiones} siguientes:

Las conclusiones a las que se han llegado en este trabajo de investigación son las 
Hipótesis H1. Los alumnos que han elegido ciencias tienen un autoconcepto académico, social, físico, familiar, emocional y un rendimiento académico más alto que aquellos alumnos que han elegido letras. No se confirma esta hipótesis al 100\%, ya que los alumnos que han elegido ciencias obtienen una media más alta en los autoconceptos académico, social y familiar frente a los alumnos que han elegido letras. Pero en el autoconcepto físico y emocional, esto no se cumple y son los alumnos que han elegido letras los que presentan el autoconcepto más alto.

Hipótesis H2. Los alumnos que tienen un autoconcepto total alto han elegido ciencias. No se confirma esta hipótesis, ya que el $81,8 \%$ de los alumnos con un autoconcepto total alto eligen letras frente al $77,8 \%$ de los alumnos con un autoconcepto total alto y que eligen ciencias.

Hipótesis H3. Las mujeres tienen un autoconcepto en todas sus dimensiones más alto que los hombres. Esta hipótesis solo se cumple en el caso del autoconcepto académico, donde el $72,7 \%$ de las mujeres tienen un este autoconcepto alto frente $44,5 \%$ de los hombres. En el resto de autoconceptos (social, físico, familiar y emocional) los hombres tienen un nivel de autoconcepto más alto que las mujeres.

Hipótesis H4. Los alumnos con un autoconcepto total alto tienen un rendimiento académico alto. Esta hipótesis queda confirmada. Los datos nos dicen que el 15\% de los alumnos con un autoconcepto total bajo tienen un nivel académico de "Aprobado", mientras que el $30 \%$ de los alumnos con un autoconcepto total alto tienen un nivel académico de "Aprobado". En el mismo sentido, el 5\% de los alumnos con un autoconcepto total bajo tienen un nivel académico de "Notable" y el 30\% de los alumnos con un autoconcepto total alto tienen un nivel académico de "Notable". Por último, no hay ningún alumno con autoconcepto total bajo que haya obtenido un nivel académico de "Sobresaliente" mientras que el 5\% de los alumnos con autoconcepto total alto tienen un nivel académico de "Sobresaliente".

Las conclusiones más importantes a las que se llegaron fueron que los alumnos con mayor autoconcepto académico tienen un rendimiento académico alto y la opción preferida por los alumnos con mayor rendimiento académico es la de ciencias. En cuanto al género, las mujeres tienen un mayor autoconcepto académico que los hombres. Además, no podemos decir que exista una relación entre el género y la opción elegida.

La principal limitación que se encontró en la investigación fue el número de alumnos participantes en la misma, ya que el colegio solo disponía de una clase de $3^{\circ}$ de ESO con 23 alumnos. Otra de las limitaciones que se encontraron, relacionada con el número de alumnos, fue que 3 de los 23 alumnos participantes en la investigación repitieron de curso, por lo que no pudieron elegir entre ciencias o letras. Se realizó al colegio una propuesta de continuidad en el tiempo sobre este estudio para poder corroborar los datos obtenidos en el mismo. Además, se propone incluir en nuestro análisis herramientas que nos permitan incluir o ahondar en los aspectos que influyen en la elección de los alumnos.

\section{Referencias}

Alcaide, M. (2009). Influencia del rendimiento y autoconcepto en hombres y mujeres. Revista Electrónica de Investigación y Docencia, 2, 27-44. 
Bautista, C. (s.f.). monografías.com. Recuperado de http://www.monografias.com/trabajos81/caracteristicas-adolescente-proceso crecimiento/caracteristicas-adolescente-proceso-crecimiento2.shtml.

Brooks-gunn, J., J.A. Graber, and R.L. Paikoff (1994). Studying links between hormones and negative affect: models and measures. Journal of Research on Adolescence, 4(4), 469-486.

Buchanan, C.M., Maccoby, E.E., and Dornbusch, S.M. (1992). Adolescents and their families after divorce: three residential arrangements. Journal of Research on Adolescence, 2, 261-291

Castaño, C. (1983). Psicología y orientación vocacional. Madrid: Marova

Crain, M. (1996). The influence of age, race and gender on child and adolescent selfconcept. En B. A. Bracken (Ed.), Handbook of self-concept (pp. 395-420). New York: Wiley.

Chambers, R.A., Taylor, J.R., \& Potenza, M.N. (2003). Developmental neurocircuitry of motivation in adolescence: Acritical period of addiction vulnerability. American Journal of Psychiatry, 160, 1041-1052.

García, M., González-Pumariega, S., Roces, C., Álvarez, L. y González, M. d. (1998). Estrategias de Aprendizaje, Autoconcepto y Rendimiento Académico. Psicothema, 10(1), 97-109.

García, F. y Musitu, G. (2001). Manual de autoconcepto Forma 5. Madrid: TEA

Edel, R. (2003). El rendimiento académico: concepto, investigación y desarrollo. Revista Electrónica Iberoamericana sobre Calidad, Eficacia y Cambio en Educación, 1(2)

Eliana, M., Villalobos, J. y Astrid, V. (2007). Factores institucionales, pedagógicos, psicosociales y sociodemográficos asociados al rendimiento académico en la Universidad de Costa Rica: un análisis multinivel. Revista Electrónica de Investigación y Evaluación Educativa, 13(2), 215-234.

González, J. A. (2003). El rendimiento escolar. Un análisis de las variables que lo condicionan. Revista Galagico-portuguesa de Psicoloxía e Educación, 8(7),247258.

González, J. A., J.Carlos, N., Gonzáles-Pumariega, S., \& García, M. S. (1997). Autoconcepto, Autoestima y Aprendizaje Escolar. Psicothema, 9(2), 271-289.

González, M. d., \& Tourón, J. (1992). Autoconcepto y Rendimiento escolar. Pamplona: Ediciones Universidad de Navarra, S.A.

Goñi, E., Ruiz de Azúa, S. y Goñi, A. (2007). Autoconcepto personal y satisfacción con la vida. International Journal of the Developmental and Educational Psychology, 1(2) 187-198.

Harter, S. (1990). Issues in the assessment of the self-concept of children and adolescents. En A.M. La Greca (ed.), Through the eyes of the child: Obtaining self-reports from children and adolescents (pp. 292-325). Boston: Allyn \& Bacon.

Jiménez, M. (2000). Competencia social: intervención preventiva en la escuela. Infancia y Sociedad, 24, 21-48. 
Kalish, R. (1983). La vejez: Perspectivas sobre el Desarrollo Humano. Madrid: Pirámide.

Latiesa, M. (1989). Demanda de Educación Superior: evaluaciones y condicionamientos de los estudiantes en la elección de carrera. Reis, 101-139.

López-Barajas (1985). Didáctica: metodología de la investigación.Madrid.UNED

Miller, P. (1979). Sex of subject and self-concept variables. En R.C. Wylie (ed.), The self- concept, 2, 241-328.

Mosteiro, M. J. (1997). El género como factor condicionante de la elección de carrera: hacia una orientación para la igualdad de oportunidades entre los sexos. Revista galego-portuguesa de psicoloxía e educación, 305-315.

Molero, D., Zagalaz, M.L. y Cachón, J. (2013). Estudio comparativo del autoconcepto físico a lo largo del ciclo vital. Revista de Psicología del Deporte, 22(1), 135142.

Oliva, A. (2006). Relaciones familiares y desarrollo adolescente. Anuario de Psicología, 37(3), 209.

Osypow, S. (1987). Couseling Psychology: Theory, research and practice in career couseling. Annual Review of Psychology, 38, 257-278.

Padilla, M, ( 2015). Autoconcepto en adolescentes de 13 a 15 años de educación general básica. Tesis doctoral. Recuperado de: http://dspace.ucuenca.edu.ec/handle/123456789/21942

Palomo, M. (2017). El autoconcepto y la motivación escolar: una revisión bibliográfica. Revista INFAD de Psiclología. Recuperado de http://www.infad.eu/RevistaINFAD/OJS/index.php/IJODAEP/article/view/737

Piaget, J. (1973). Psicología del niño. Madrid: Morata

Riquelme, E y Riquelme, P. (2011) Análisis Psicométrico confirmatorio de la medida multidimensional del Test de Autoconcepto Forma 5 en Español (AF5), en estudiantes universitarios de Chile. Recuperado dehttp://www.scielo.mec.pt/scielo.php?pid=S164500862011000100006\&script= sci_arttext\&tlng=en

Robledo, P., y García, J.-N. (2009). El entorno familiar y su influencia en el rendimiento académico de los alumnos con dificultades de aprendizaje: revisión de estudios empíricos. Aula Abierta, 37(1), 117-128.

Rodríguez, M. d., Peña, J. V., \& Inda, M. d. (2016). Esto es lo que me gusta y lo qye voy a estudiar: Un estudio cualitativo sobre la toma de decisiones académicas en bachillerato. Revista Complutense de Educación, 27(3), 1351-1368.

Rodríguez, R (2014). Autoestima y rendimiento académico: un estado de la cuestión. Recuperado de http://reunir.unir.net/handle/123456789/2615

Royo, M.A. y Jiménez Correa, A. (1993). Comunicación y educación. Revista de educación de la Universidad de Granada, 12, 113-125.

Smetana, J. G. (1989). Adolescents' and parents' reasoning about actual family conflict. Child Development, 60, 1052-1067. 
Tomás, J. M., \& Oliver, A. (2004). Análisis psicométrico confirmatorio de una medida multidimensional del autoconcepto en español. Interamerican Journal of Psychology , 38, 285-294.

Fecha de recepción: 19/04/2017

Fecha de revisión: 29/05/2017

Fecha de aceptación: 31/10/2017 